\title{
Electro-optic comb pumped optical parametric oscillator with flexible repetition rate at $\mathrm{GHz}$ level
}

\author{
Hanyu Ye ${ }^{1,+},{ }^{*}$, Valerian Freysz ${ }^{2, \dagger}$, Ramatou Bello-Doua ${ }^{3}$, Lilia Pontagnier ${ }^{1}$, Giorgio \\ SANTARELli ${ }^{1}$, ERIC Cormier ${ }^{1,4}$, ANd Eric Freysz ${ }^{2}$ \\ ${ }^{1}$ Laboratoire Photonique Numérique et Nanosciences (LP2N), UMR 5298, CNRS-IOGS-Université Bordeaux, 33400 Talence, France \\ ${ }^{2}$ Université de Bordeaux, CNRS, LOMA, UMR 5798, 33400 Talence, France \\ ${ }^{3}$ ALPhANOV, Institut d'optique d'Aquitaine, Rue François Mitterrand, 33400 Talence, France \\ ${ }^{4}$ Institut Universitaire de France (IUF), 1 rue Descartes, 75231 Paris, France \\ ${ }^{+}$These authors contributed equally to this work \\ * Corresponding author: hanyu.ye@institutoptique.fr
}

Compiled March 24, 2021

We present a gigahertz (GHz)-repetition-rate optical parametric oscillator (OPO) pumped by an electro-optic (EO) comb at $1.03 \mu \mathrm{m}$, delivering sub-picosecond signal pulses across 1.5-1.7 $\mu \mathrm{m}$ from a $\mathrm{MgO}$-doped periodically poled $\mathrm{LiNbO}_{3}$ crystal. Using a pump power of 5 $\mathrm{W}$ at $14.2 \mathrm{GHz}$ repetition rate, $378 \mathrm{~mW}$ of signal power is obtained at $\mathbf{1 . 5 2} \mu \mathrm{m}$ from a subharmonic cavity, corresponding to a signal extraction efficiency of $7.6 \%$. By cascading a Mach-Zehnder modulator, the pump pulse repetition rate can be divided by any integer number from 1 to 14, allowing the OPO to operate with flexible repetition rate from 1 to $14.2 \mathrm{GHz}$. Besides, a strategy leading to quasi-continuous repetition rate tunability of the OPO is also discussed. (- 2021 Optical Society of America

http://dx.doi.org/10.1364/ao.XX.XXXXXX

Electro-optic (EO) frequency combs are attracting more and more attentions as a new kind of ultrafast laser sources with $\mathrm{GHz}$ repetition rates [1-4]. Such high-repetition-rate lasers have great potentials in various fields, such as advanced material processing [5], ultrafast time-domain spectroscopy [6], free-space optical communication [7] and electron guns [8]. Compared to traditional mode-locked lasers, the repetition rate scaling of an EO comb in single-pass configuration is not bound to the cavity length of the laser, enabling easy repetition rate selection in a wide $\mathrm{GHz}$ range using a radiofrequency (RF) synthesizer. The naturally chirped pulses emerging from the EO combs also make it convenient to integrate with all-fiber chirped-pulse amplifiers (CPAs). Besides, the line spacing of an EO comb is inherently stable, and the carrier-envelope offset frequency is clearly defined by a single-frequency seed laser. Relying on mature single-frequency diode lasers, $\mathrm{Yb}$ - and Er-doped fiber amplifiers and commercially available EO modulators (EOMs), ultrafast $\mathrm{GHz} \mathrm{EO}$ combs have been mainly demonstrated at $\sim 1$ and $\sim 1.5 \mu \mathrm{m}$. Efforts on converting these EO combs to other wavelengths for maximizing their potentials are still rare. Regarding frequency up-conversion, a multi-GHz repetition rate deep ultraviolet source in burst mode derived from an $\mathrm{EO}$ comb at $1.03 \mu \mathrm{m}$ [9] and a broadband $30 \mathrm{GHz}$ EO comb in the visible and near-infrared (near-IR) [10] have been reported. Regarding frequency down-conversion for mid-IR pulse generation, $10 \mathrm{GHz}$ frequency combs covering 3.1-4.8 $\mu \mathrm{m}$ and 7-11 $\mu \mathrm{m}$ by intra-pulse difference-frequency generation (DFG) of a spectrally broadened EO comb centered at $1.55 \mu \mathrm{m}$ have been demonstrated [11]. Besides, microresonators driven by EO combs have also shown potentials for harmonic generation at short wavelengths and optical parametric oscillation in the mid-IR [12]. Nevertheless, for generating powerful, high-beam-quality and ultrashort pulsed radiations tunable in the near- and mid-IR, synchronously pumped optical parametric oscillators (OPOs) based on bulk nonlinear crystals are still a promising approach.

Previously, synchronously pumped OPOs with high repetition rates of tens of $\mathrm{GHz}$ have been demonstrated [13-15]. However, such high-repetition-rate operation requires challenging cavity designs for the driving mode-locked lasers or very high order harmonic mode-locking technique [16], which are usually accompanied with multi-picosecond pulses. More often, multi-GHz repetition rates of synchronously pumped OPOs are achieved by using a conventional mode-locked laser at tens or hundreds of $\mathrm{MHz}$ repetition rate together with a harmonic OPO cavity whose length is proportional to the pump laser cavity length $[17,18]$. Although this method could easily and flexibly extend the repetition rate up to multiple $\mathrm{GHz}$, the uneven envelop of the output pulse train is always inevitable due to cavity loss of the resonant pulses in each round trip before being synchronized with the next pump pulse. This limit prevents its practicality for many applications.

In this letter, based on recent development of $\mathrm{EO}$ combs, we demonstrate an ultrafast EO comb pumped OPO delivering multi-GHz pulses. The 1.03- $\mu \mathrm{m} \mathrm{EO}$ comb system delivers $5 \mathrm{~W}$ of average power and $1.16 \mathrm{ps}$ de-chirped pulses at $14.2 \mathrm{GHz}$ repetition rate. The comb pumped OPO generates sub-picosecond signal pulses up to $378 \mathrm{~mW}$ at $1.52 \mu \mathrm{m}$ with the capability of covering the 1.5-1.7 $\mu \mathrm{m}$ spectral band by crystal temperature tuning. An additional Mach-Zehnder Modulator (MZM) is further used as a divider to realize flexible repetition rate operation from 1 to $14.2 \mathrm{GHz}$. Furthermore, a strategy for obtaining quasi- 


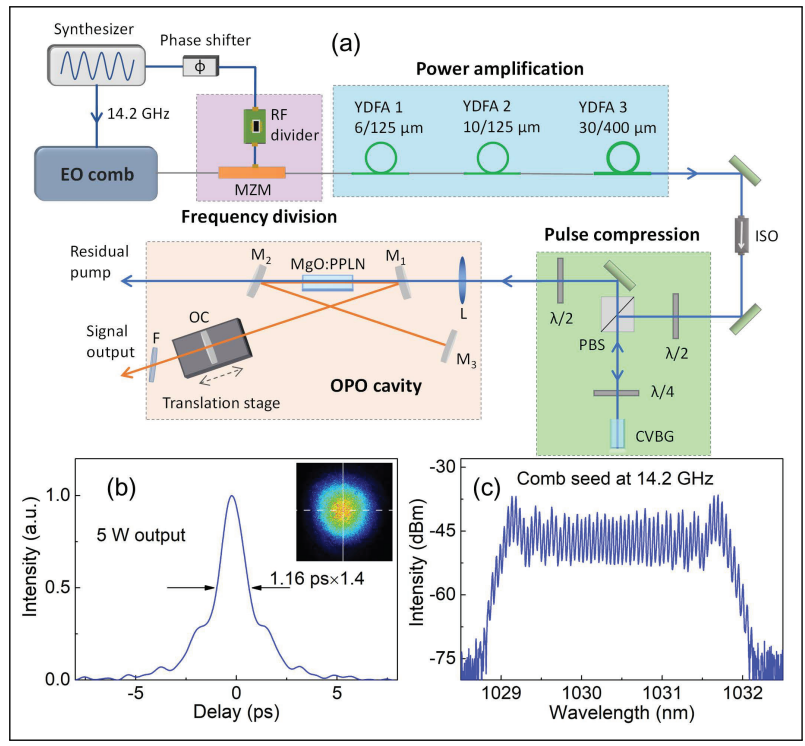

Fig. 1. (a) Experimental setup of the EO comb pumped OPO with multi-GHz repetition rates. MZM, Mach-Zehnder Modulator; YDFA, Yb-doped fiber amplifier; ISO, isolator; $\lambda / 2$, half-wave plate; $\lambda / 4$, quarter-wave plate; PBS, polarizingbeam splitter; CVBG, chirped volume Bragg grating; $L$, lens; M, mirror; OC, output coupler; F, filter. (b) Autocorrelation of the $\mathrm{EO}$ comb at $5 \mathrm{~W}$ after compression. Inset: Beam profile of the EO comb at $5 \mathrm{~W}$ after compression. (c) Measured spectrum of the EO comb seed at $14.2 \mathrm{GHz}$.

continuous repetition rate tunability is also discussed.

The experimental setup for the EO comb pumped OPO with multi-GHz repetition rates is shown in Fig.1(a). The EO comb originating from a continuous-wave single-frequency diode laser at $1.03 \mu \mathrm{m}$ consists of two phase EOMs for spectral broadening and a MZM for pulse formation and linear chirp selection, as detailed in [2]. Another MZM sharing the same RF synthesizer is further used to divide the pulse repetition rate. Frequency division by any integer number from 1 to 14 is enabled by using a binary-controlled RF divider. The power of the divided pulses varies from $1 \mathrm{~mW}$ to $38 \mathrm{~mW}$, depending on the division factor, and is boosted by three cascaded $\mathrm{Yb}$-doped fiber amplifiers (YDFAs). The first stage is a core-pumped YDFA $(6 / 125 \mu \mathrm{m})$, providing 50-102 $\mathrm{mW}$ output power correspondingly. The second and final stages are cladding-pumped YDFAs $(10 / 125 \mu \mathrm{m}$ and $30 / 250 \mu \mathrm{m}$ ), providing $\sim 800 \mathrm{~mW}$ and $>7 \mathrm{~W}$ output powers, respectively. The amplified comb is collimated to free space and guided through an isolator to prevent optical feedback. Then we employ a chirped volume Bragg grating (CVBG) based compressor to de-chirp the comb pulses. In the compressor, a halfand quarter-wave plate together with a polarizing-beam splitter (PBS) are used to inject the comb pulses into the CVBG and then extract the reflected beam for pumping the OPO. Since the compressor only provides a fixed dispersion, we adjust the driving RF frequency of the EO comb and thus the chirp amount in the modulated pulses to find the RF frequency where the compressor perfectly cancels the chirp of the comb pulses. By monitoring the autocorrelation (AC) of the compressed pulses, we confirm the optimal frequency to be around 14.2 GHz. Considering the compressor efficiency of $\sim 80 \%$ and optical loss in the isolator, $\sim 5 \mathrm{~W}$ of comb power are available to drive the OPO.

In the frequency conversion part, the $\mathrm{EO}$ comb is corrected to vertical polarization and focused into a 10-mm-long $\mathrm{MgO}-$ doped periodically poled $\mathrm{LiNbO}_{3}$ (MgO:PPLN) crystal with a beam waist radius of $\sim 49 \mu \mathrm{m}$ for achieving phase matching and cavity mode matching. The MgO:PPLN crystal housed in an oven contains five different grating periods with $29.98 \mu \mathrm{m}$ and $30.49 \mu \mathrm{m}$ used for the OPO. We use an X-cavity with a length of $\sim 590 \mathrm{~mm}$, corresponding to a free spectral range (FSR) of $\sim 249 \mathrm{MHz}$ and 57 signal pulses circulating in the cavity at 14.2 $\mathrm{GHz}$ repetition rate. The cavity consists of two curved mirrors (from Layertec Inc.), $\mathrm{M}_{1}$ and $\mathrm{M}_{2}$ ( $\mathrm{r}=100 \mathrm{~mm}$ ), anti-reflection (AR) coated around $1.03 \mu \mathrm{m}(\mathrm{R}<2 \%)$ and high reflection (HR) coated for 1.4-2.1 $\mu \mathrm{m}(\mathrm{R}>99.8 \%)$, as well as a plane mirror $\mathrm{M}_{3}$ with the same HR coating and an output coupler (OC) with $\sim 2 \%$ transmission across $1.5-1.7 \mu \mathrm{m}$, ensuring singly resonant operation for the signal wave. The two curved mirrors made of fused silica are not coated for the mid-IR idler. Thus, the OPO characterization is mainly based on the near-IR signal. Finally, for synchronization between the comb and signal pulses, the $\mathrm{OC}$ is placed on a high-precision linear translation stage and followed by a long-pass filter with a cut-on wavelength of 1200 $\mathrm{nm}$ to block visible parasitic light.

We first characterized the EO comb before pumping the OPO. Figure 1(b) shows the AC trace of the comb pulses after compression at $14.2 \mathrm{GHz}$. Considering a deconvolution factor of 1.4 (inherent to $\mathrm{EO}$ comb generated pulses), the pulse duration is $1.16 \mathrm{ps}$ at $5 \mathrm{~W}$. The spatial profile of the $\mathrm{EO}$ comb at $5 \mathrm{~W}$ is also captured using a CCD camera, indicating a single-mode distribution, as shown in the inset of Fig. 1(b). The spectrum of the EO comb seed at $14.2 \mathrm{GHz}$ is measured and plotted in Fig. 1(c). The modulated spectrum has a $10 \mathrm{~dB}$ bandwidth of $\sim 2.5$ $\mathrm{nm}$ and a signal-to-noise ratio (SNR) of $36 \mathrm{~dB}$ measured with a resolution of $0.02 \mathrm{~nm}$. After amplification, the final spectrum has no significant change except that the profile becomes slightly tilted due to the limited gain bandwidth of the used YDFAs, which is centered at a longer wavelength.

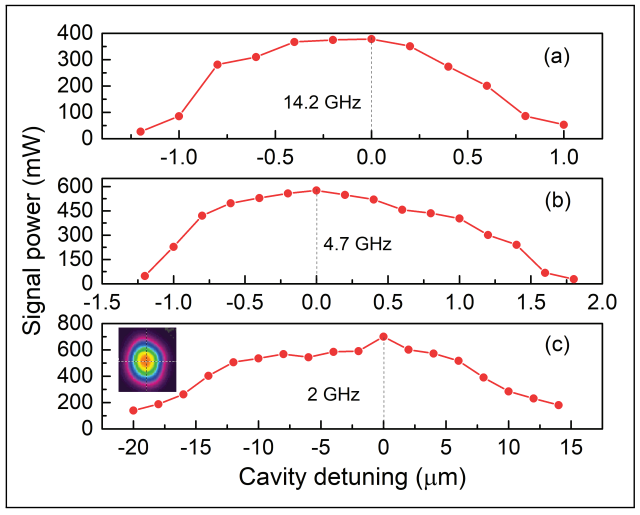

Fig. 2. Output signal powers as functions of cavity detunings at (a) $14.2 \mathrm{GHz}$, (b) $4.7 \mathrm{GHz}$ and (c) $2 \mathrm{GHz}$ with $5 \mathrm{~W}$ pump power. Inset: Beam profile of the signal at $2 \mathrm{GHz}$ and $700 \mathrm{~mW}$.

Using this $5 \mathrm{~W}$ EO comb as pump, we further implemented the OPO. Initially, we kept the MgO:PPLN crystal at $50^{\circ} \mathrm{C}$ and used the grating period of $29.98 \mu \mathrm{m}$, corresponding to a signal wavelength of $1.52 \mu \mathrm{m}$. At the fundamental repetition rate of 14.2 GHz, the OPO threshold was observed for $\sim 3 \mathrm{~W}$ of pump power. Then, we recorded the output signal power at different cavity detuning values. The OPO allows a synchronization range of $2.2 \mu \mathrm{m}$ and delivers a maximum signal power of 378 $\mathrm{mW}$, corresponding to a signal extraction efficiency of $7.6 \%$, as 


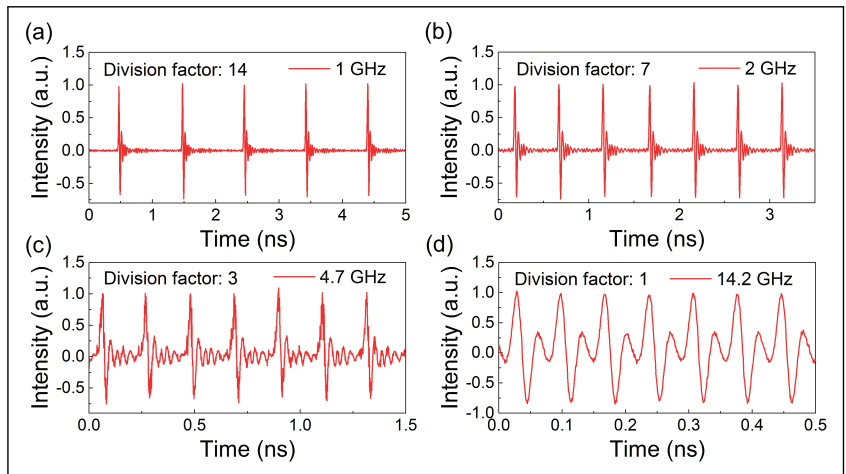

Fig. 3. Measured pulse trains of the OPO signal at different repetition rates: (a) $1 \mathrm{GHz}$, (b) $2 \mathrm{GHz}$, (c) $4.7 \mathrm{GHz}$ and (d) 14.2 $\mathrm{GHz}$.

shown in Fig. 2(a). For comparison, fig. 2(b) and (c) show the same measurement at divided repetition rates of $4.7 \mathrm{GHz}$ and 2 $\mathrm{GHz}$, corresponding to division factors of 3 and 7, respectively. With three times higher pump pulse energy, the OPO allows a larger synchronization range of $3 \mu \mathrm{m}$ at $4.7 \mathrm{GHz}$ and delivers a higher signal power of $576 \mathrm{~mW}$ at zero detuning. At $2 \mathrm{GHz}$ with seven times higher pump pulse energy, the obtained synchronization range reaches $34 \mu \mathrm{m}$, much larger than the previous two cases, but the output signal power only increases to 700 $\mathrm{mW}$, implying the OPO enters a saturation regime where backconversion and signal pulse breakdown [19] have occurred due to high pulse energy circulating in the cavity. Besides, we also captured a single-mode profile of the signal beam at $700 \mathrm{~mW}$ using a pyroelectric camera, as shown in the inset of Fig. 2(c).

By fully exploiting the frequency division function, we could divide the $14.2 \mathrm{GHz}$ repetition rate by any integer number up to 14, making the EO comb highly flexible for repetition rate selection with almost constant output power and pulse duration after compression, with the assistance of the fiber CPA system which avoids excess nonlinear effects. When pumping the OPO at different divided repetition rates, there are always subharmonic cavity FSRs near $249 \mathrm{MHz}$. Thus, by just adjusting the cavity length, we could obtain the corresponding FSRs and operate the OPO across 1-14.2 GHz. Using a fast InGaAs photodiode (Optilab, PD-40x) with a bandwidth of $40 \mathrm{GHz}$ and a sampling oscilloscope (Keysight, DCA-X 86100D) with a bandwidth of $45 \mathrm{GHz}$, we measured the signal pulse trains at various repetition rates. Figures 3(a)-(d) display four recorded traces at 1.01 $\mathrm{GHz}, 2.03 \mathrm{GHz}, 4.73 \mathrm{GHz}$ and $14.2 \mathrm{GHz}$. They correspond to division factors of 14, 7, 3 and 1, respectively. As can be seen, when increasing the OPO repetition rate, the pulse structures are getting close to the bandwidth limit of the measuring equipment. Besides, the negative values of the detected pulses followed by decaying oscillations are electronic response of the photodiode to ultrashort pulses, which are not present in the AC traces.

At the fundamental repetition rate of $14.2 \mathrm{GHz}$, we further measured the AC traces of the signal pulses at different cavity detunings, as shown in Fig. 4(a). With a positive cavity detuning of $+500 \mathrm{~nm}$, the FWHM duration of the AC trace is 830 fs. With cavity detunings of zero and $-800 \mathrm{~nm}$, the AC durations increased to $980 \mathrm{fs}$ and $1 \mathrm{ps}$, respectively. Compared to the pump, the generated signal pulses have shorter durations and cleaner profiles due to the filtering effect of this nonlinear process. Figure 4(b) depicts the corresponding measured spectra. For $+500 \mathrm{~nm}$ detuning, the signal spectrum is centered at 1524

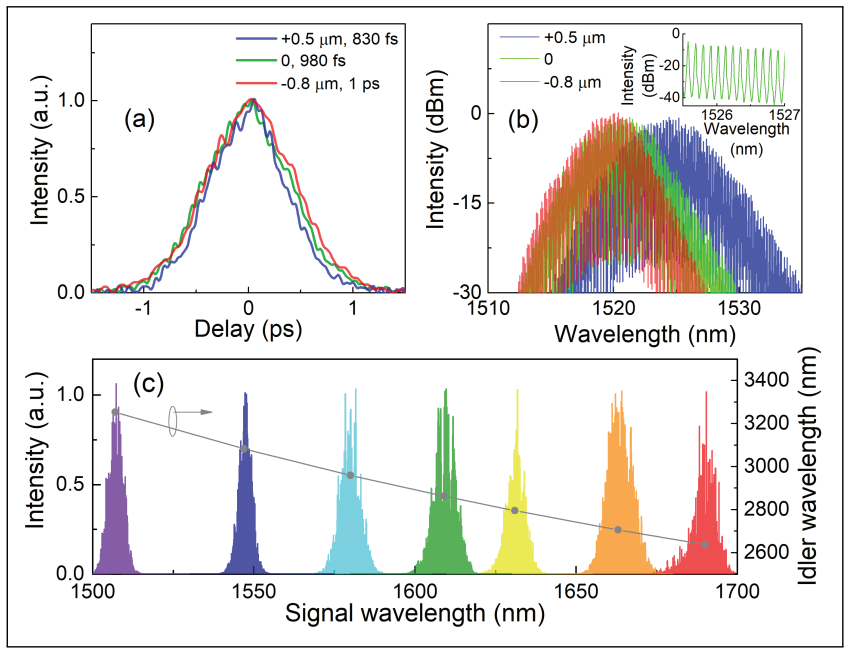

Fig. 4. (a) Autocorrelations and (b) spectra of the signal pulses at $14.2 \mathrm{GHz}$ with different cavity detunings. (c) Measured signal spectra tunable across 1.5-1.7 $\mu \mathrm{m}$ and the corresponding calculated idler wavelengths.

$\mathrm{nm}$ and has a $10 \mathrm{~dB}$ bandwidth of $10.7 \mathrm{~nm}$. For zero and -800 $\mathrm{nm}$ detunings, the signal spectrum is blue-shifted to $1520.5 \mathrm{~nm}$ and $1520 \mathrm{~nm}$, and has a $10 \mathrm{~dB}$ bandwidth of $9.1 \mathrm{~nm}$ and 7.8 $\mathrm{nm}$, respectively. Fourier transform of these spectra indicates that the measured AC traces are only 1.08, 1.1 and 1.03 times their transform limits for $+500 \mathrm{~nm}, 0$ and $-800 \mathrm{~nm}$ detunings, respectively. The above signal evolution can be explained as follows. With positive cavity detunings, the signal pulses arrive behind the pump pulses at the entrance of the MgO:PPLN crystal and catch up during propagation in the crystal with a GVM of $\sim 0.1 \mathrm{ps} / \mathrm{mm}$. The latter leads to relatively uniform amplification for the whole temporal and spectral profiles of the signal pulses. At contrast, with negative cavity detunings, the pump pulses are mainly overlapped with the trailing wings of the signal pulses and these trailing wings are dragged and prolonged by the pump pulses due to GVM in the crystal. Besides, considering that the crystal provides positive dispersion for the signal, the signal trailing wings containing higher optical frequencies experience more parametric gain than the leading part. This leads to narrower and blue-shifted signal spectra, as well as near transform-limited pulses.

Similarly, at $4.7 \mathrm{GHz}$, with $5 \mathrm{~W}$ of pump power, we observed identical temporal and spectral behaviours of the signal. However, at $2 \mathrm{GHz}$ with seven times higher pump energy, strong pedestals appear on the signal AC trace around zero cavity detuning. This indicates that the signal pulse breakdown into a few satellite pulses, which could increase the overlap range between the pump and signal pulses and thus explain the much larger synchronization range in Fig. 2(c). Similar to [19], by reducing the pump power, we managed to eliminate the pulse breakdown effect. Another mitigation method could be using another OC with a higher signal transmission.

At $14.2 \mathrm{GHz}$, we also performed coarse tuning measurement for the signal wavelength. Using the grating periods of 29.98 $\mu \mathrm{m}$ and $30.49 \mu \mathrm{m}$ in the crystal together with the temperature varying from room temperature up to $150^{\circ} \mathrm{C}$, we achieved a signal output tunable across 1.5-1.7 $\mu \mathrm{m}$, as shown in Fig. 4(c). Despite the lack of proper optics for extracting the mid-IR idler, we calculated the generated idler wavelength, which was tun- 


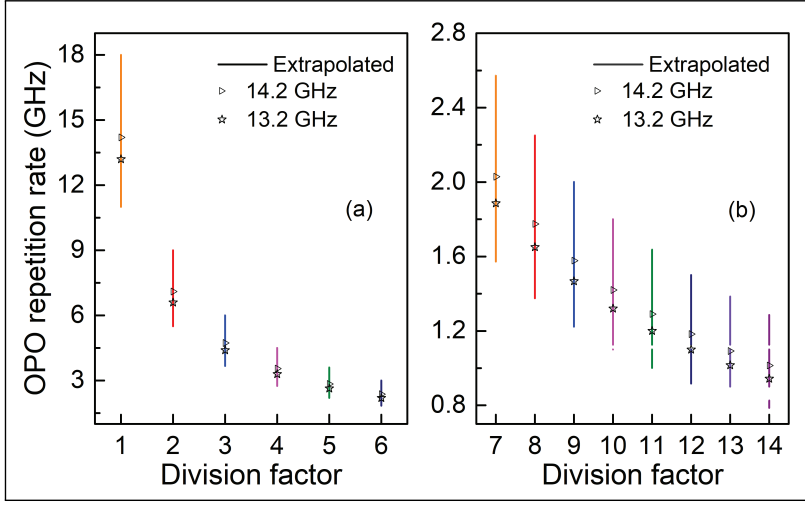

Fig. 5. Demonstrated OPO repetition rates and extrapolated ranges assuming using a diffraction grating based compressor at division factors of (a)1-6 and (b) 7-14.

able across 2.64-3.25 $\mu \mathrm{m}$, based on photon energy conservation, as also shown in Fig. 4(c).

Last, to highlight the potential of our system for generating extremely flexible repetition rates, we calculated the OPO repetition rates which could be achieved by tuning the fundamental frequency of the EO comb and adjusting the cavity FSR. In the calculation, we considered the tuning range of the fundamental frequency of the EO comb to be 11-18 GHz which has been demonstrated previously [2] and a $\pm 10 \%$ FSR variation around $250 \mathrm{MHz}$, within which the cavity remains stable. The corresponding extrapolated OPO repetition rates at each division factor are displayed in Fig. 5(a) and (b). As can be seen, this concept allows the OPO to cover a wide quasi-continuous repetition rate range of $0.8-18 \mathrm{GHz}$ however interrupted between 9 and $11 \mathrm{GHz}$ and with small gaps for division factors greater than 9. In the experiment, since we are using a compressor providing a fixed dispersion, we have limited the fundamental frequency tuning to $1 \mathrm{GHz}$ (14.2 to $13.2 \mathrm{GHz}$ ), where the pulse duration of the EO comb after compression remains shorter than $1.35 \mathrm{ps}$. The corresponding divided repetition rates were also achieved and plotted in Fig. 5. Note here that using a diffraction grating based compressor would allow to maintain short pulses across 11-18 GHz [2], and thus allowing the OPO to operate on the much wider repetition rate range.

In conclusion, we have demonstrated an ultrafast OPO with flexible repetition rate across 1-14.2 GHz pumped by an EO comb at $1.03 \mu \mathrm{m}$. At $14.2 \mathrm{GHz}$, up to $378 \mathrm{~mW}$ of signal power at $1.52 \mu \mathrm{m}$ is obtained with $5 \mathrm{~W}$ pump power. A wavelength tunability over 1.5 to $1.7 \mu \mathrm{m}$ is further achieved by changing the temperature of the MgO:PPLN crystal. Combining the frequency division technique and cavity length scanning of the high subharmonic OPO, we could operate the OPO at flexible repetition rates from 1 to $14.2 \mathrm{GHz}$. Furthermore, by including the concept of adjusting the fundamental frequency of the EO comb as well as the cavity FSR, we show that the OPO repetition rate could be potentially highly tunable. The demonstration of our OPO system paves the way for further development of GHz-repetition-rate sources tunable in the near- and mid-IR regions. Besides, it also provides a new solution for mid-IR multi-GHz frequency comb generation with additional cavity locking systems.

Funding. Agence Nationale de la Recherche (ANR-10-IDEX-0302); Institut Universitaire de France; Aquitaine Science transfert (AST_AT_2018-043); H2020 LEIT Information and Communication Tech- nologies (H2020-ICT-2018); Conseil Régional Nouvelle Aquitaine (20191R5M04).

Acknowledgments. The authors acknowledge financial supports from the French National Research Agency (ANR) in the frame of "the investments for the future" Program IdEx Bordeaux - LAPHIA (ANR-10IDEX-03-02), the Institut Universitaire de France, and European Union's Horizon 2020 research and innovation programme under grant agreement No 825246 (Flexiburst).

Disclosures. The authors declare no conflicts of interest.

\section{REFERENCES}

1. D. R. Carlson, D. D. Hickstein, W. Zhang, A. J. Metcalf, F. Quinlan, S. A. Diddams, and S. B. Papp, Science 361, 1358 (2018).

2. A. Aubourg, J. Lhermite, S. Hocquet, E. Cormier, and G. Santarelli, Opt. Lett. 40, 5610 (2015).

3. A. Parriaux, K. Hammani, and G. Millot, Adv. Opt. Photon. 12, 223 (2020).

4. X. Zhang, J. Zhang, K. Yin, Y. Li, X. Zheng, and T. Jiang, Opt. Express 28, 34761 (2020).

5. S. Schwarz, S. Rung, C. Esen, and R. Hellmann, Opt. Lett. 46, 282 (2021).

6. R. Gebs, G. Klatt, C. Janke, T. Dekorsy, and A. Bartels, Opt. Express 18, 5974 (2010).

7. Y. Su, W. Wang, X. Hu, H. Hu, X. Huang, Y. Wang, J. Si, X. Xie, B. Han, H. Feng, Q. Hao, G. Zhu, T. Duan, and W. Zhao, Opt. Express 26, 34515 (2018).

8. C. Li, L. Winkelmann, and I. Hartl, "6 ghz repetition rate photocathode laser for multi-bunch operation of a relativistic electron gun," in Conference on Lasers and Electro-Optics, (Optical Society of America, 2018), p. SF3N.1.

9. H. Ye, L. Pontagnier, C. Dixneuf, G. Santarelli, and E. Cormier, Opt. Express 28, 37209 (2020).

10. A. J. Metcalf, C. D. Fredrick, R. C. Terrien, S. B. Papp, and S. A. Diddams, Opt. Lett. 44, 2673 (2019).

11. A. S. Kowligy, D. R. Carlson, D. D. Hickstein, H. Timmers, A. J. Lind, P. G. Schunemann, S. B. Papp, and S. A. Diddams, Opt. Lett. 45, 3677 (2020).

12. S. J. Herr, V. Brasch, J. Szabados, E. Obrzud, Y. Jia, S. Lecomte, K. Buse, I. Breunig, and T. Herr, Opt. Lett. 43, 5745 (2018).

13. S. Lecomte, L. Krainer, R. Paschotta, M. J. P. Dymott, K. J. Weingarten, and U. Keller, Opt. Lett. 27, 1714 (2002).

14. S. Lecomte, R. Paschotta, S. Pawlik, B. Schmidt, K. Furusawa, A. Malinowski, D. J. Richardson, and U. Keller, Opt. Lett. 30, 290 (2005).

15. S. Lecomte, R. Paschotta, S. Pawlik, B. Schmidt, K. Furusawa, A. Malinowski, D. J. Richardson, and U. Keller, IEEE Photonics Technol. Lett. 17, 483 (2005).

16. H. Chen, S. Chen, Z. Jiang, and J. Hou, Opt. Express 23, 1308 (2015).

17. O. Kokabee, A. Esteban-Martin, and M. Ebrahim-Zadeh, Opt. Express 17, 15635 (2009).

18. A. Aadhi, V. Sharma, N. A. Chaitanya, and G. Samanta, Sci. Rep. 7, 43913 (2017).

19. L. Lefort, K. Puech, S. D. Butterworth, Y. P. Svirko, and D. C. Hanna, Opt. Lett. 24, 28 (1999). 


\section{FULL REFERENCES}

1. D. R. Carlson, D. D. Hickstein, W. Zhang, A. J. Metcalf, F. Quinlan, S. A. Diddams, and S. B. Papp, "Ultrafast electro-optic light with subcycle control," Science 361, 1358-1363 (2018).

2. A. Aubourg, J. Lhermite, S. Hocquet, E. Cormier, and G. Santarelli, "Generation of picosecond laser pulses at $1030 \mathrm{~nm}$ with gigahertz range continuously tunable repetition rate," Opt. Lett. 40, 5610-5613 (2015).

3. A. Parriaux, K. Hammani, and G. Millot, "Electro-optic frequency combs," Adv. Opt. Photon. 12, 223-287 (2020).

4. X. Zhang, J. Zhang, K. Yin, Y. Li, X. Zheng, and T. Jiang, "Sub-100 fs all-fiber broadband electro-optic optical frequency comb at $1.5 \mu \mathrm{m}$," Opt. Express 28, 34761-34771 (2020).

5. S. Schwarz, S. Rung, C. Esen, and R. Hellmann, "Enhanced ablation efficiency using ghz bursts in micromachining fused silica," Opt. Lett. 46, 282-285 (2021).

6. R. Gebs, G. Klatt, C. Janke, T. Dekorsy, and A. Bartels, "High-speed asynchronous optical sampling with sub-50fs time resolution," Opt. Express 18, 5974-5983 (2010).

7. Y. Su, W. Wang, X. Hu, H. Hu, X. Huang, Y. Wang, J. Si, X. Xie, B. Han, H. Feng, Q. Hao, G. Zhu, T. Duan, and W. Zhao, "10 gbps dpsk transmission over free-space link in the mid-infrared," Opt. Express 26, 34515-34528 (2018).

8. C. Li, L. Winkelmann, and I. Hartl, "6 ghz repetition rate photocathode laser for multi-bunch operation of a relativistic electron gun," in Conference on Lasers and Electro-Optics, (Optical Society of America, 2018), p. SF3N.1.

9. H. Ye, L. Pontagnier, C. Dixneuf, G. Santarelli, and E. Cormier, "Multighz repetition rate, femtosecond deep ultraviolet source in burst mode derived from an electro-optic comb," Opt. Express 28, 37209-37217 (2020).

10. A. J. Metcalf, C. D. Fredrick, R. C. Terrien, S. B. Papp, and S. A Diddams, "30 ghz electro-optic frequency comb spanning 300 thz in the near infrared and visible," Opt. Lett. 44, 2673-2676 (2019).

11. A. S. Kowligy, D. R. Carlson, D. D. Hickstein, H. Timmers, A. J. Lind, P. G. Schunemann, S. B. Papp, and S. A. Diddams, "Mid-infrared frequency combs at $10 \mathrm{ghz}$," Opt. Lett. 45, 3677-3680 (2020).

12. S. J. Herr, V. Brasch, J. Szabados, E. Obrzud, Y. Jia, S. Lecomte, $\mathrm{K}$. Buse, I. Breunig, and T. Herr, "Frequency comb up- and downconversion in synchronously driven $\chi^{(2)}$ optical microresonators," Opt. Lett. 43, 5745-5748 (2018).

13. S. Lecomte, L. Krainer, R. Paschotta, M. J. P. Dymott, K. J. Weingarten, and U. Keller, "Optical parametric oscillator with a 10-ghz repetition rate and $100-\mathrm{mw}$ average output power in the spectral region near 1.5 $\mu \mathrm{m}$," Opt. Lett. 27, 1714-1716 (2002).

14. S. Lecomte, R. Paschotta, S. Pawlik, B. Schmidt, K. Furusawa, A. Malinowski, D. J. Richardson, and U. Keller, "Optical parametric oscillator with a pulse repetition rate of $39 \mathrm{ghz}$ and 2.1-w signal average output power in the spectral region near $1.5 \mu \mathrm{m}$," Opt. Lett. 30, 290-292 (2005).

15. S. Lecomte, R. Paschotta, S. Pawlik, B. Schmidt, K. Furusawa, A. Malinowski, D. J. Richardson, and U. Keller, "Synchronously pumped optical parametric oscillator with a repetition rate of $81.8 \mathrm{ghz}$," IEEE Photonics Technol. Lett. 17, 483-485 (2005).

16. H. Chen, S. Chen, Z. Jiang, and J. Hou, "Versatile long cavity widely tunable pulsed yb-doped fiber laser with up to 27655th harmonic mode locking order," Opt. Express 23, 1308-1318 (2015).

17. O. Kokabee, A. Esteban-Martin, and M. Ebrahim-Zadeh, "Extendedcavity, tunable, ghz-repetition-rate femtosecond optical parametric oscillator pumped at $76 \mathrm{mhz}$," Opt. Express 17, 15635-15640 (2009).

18. A. Aadhi, V. Sharma, N. A. Chaitanya, and G. Samanta, "Multigigahertz, femtosecond airy beam optical parametric oscillator pumped at $78 \mathrm{mhz}$," Sci. Rep. 7, 43913 (2017).

19. L. Lefort, K. Puech, S. D. Butterworth, Y. P. Svirko, and D. C. Hanna, "Generation of femtosecond pulses from order-of-magnitude pulse compression in a synchronously pumped optical parametric oscillator based on periodically poled lithium niobate," Opt. Lett. 24, 28-30 (1999). 